\title{
Variability in the reporting of renal function endpoints in immunosuppression trials in renal transplantation: time for consensus?
}

\author{
Simon R Knight ${ }^{1,2}$ and Samia Hussain ${ }^{1,2}$ \\ ${ }^{1}$ Nuffield Department of Surgical Sciences, University of Oxford, Oxford, UK. \\ ${ }^{2}$ Centre for Evidence in Transplantation, Clinical Effectiveness Unit, Royal College of Surgeons of England, \\ London, UK
}

\section{Corresponding author:}

Mr Simon Knight MChir MA BM FRCS

Academic Clinical Lecturer

Nuffield Department of Surgical Sciences

Oxford Transplant Centre

Churchill Hospital

Old Road

Headington

OX3 7JU

Phone: +44 1865227131

E-mail: simon.knight@nds.ox.ac.uk

\section{Authorship}

SK conceived the idea for the study, extracted data, analysed data and wrote the manuscript.

$\mathrm{SH}$ extracted data, assisted in data analysis and reviewed the manuscript.

Funding

This work was supported by a grant from the Alison Hansen Foundation.

Running title: Renal function endpoints in transplant trials

Keywords: Clinical Trials, Endpoints, Graft Function, Immunosuppression, Quality, Renal Transplant.

Words: 2,898

Figures: 2

Tables: 2 


\section{Abstract}

Early measures of graft function are increasingly used to assess efficacy in clinical trials of kidney transplant immunosuppression. This study aimed to assess the variability and quality of reporting of these endpoints in contemporary trials. Data regarding renal function endpoints were extracted from 213 reports from randomised controlled trials comparing immunosuppressive interventions in renal transplant recipients published between 2010 and 2014. 174 (81.7\%) reports included a measure of renal function; in 44 (20.7\%) this was the primary endpoint. 103 manuscripts (48.4\%) reported serum creatinine, 142 (66.6\%) reported eGFR and 26 (12.2\%) reported measured GFR. Formulas used for GFR estimation were MDRD (42.3\%), Cockroft Gault (23.5\%), Nankivell (15.0\%) and CKD-EPI (0.9\%). 6 studies (2.8\%) did not report the formula used to estimate GFR. $13.9 \%$ of endpoints had missing data. In 10 studies, disagreement was found in the significance of findings using different measures of renal function. There is a great deal of variability in the reporting of renal function endpoints, with a significant proportion of studies using under-performing or inappropriate estimates. There is a need for consensus as to the best tool for monitoring and reporting renal function post-transplant, and in particular for use in clinical trials and registries. 


\section{Abbreviations}

BMI

Body Mass Index

CKD-EPI

Chronic Kidney Disease Epidemiology Collaboration

$\mathrm{CrCl}$

Creatinine Clearance

eGFR

Estimated glomerular filtration rate

GFR

Glomerular filtration rate

MDRD

Modification of Diet in Renal Disease

RCT

Randomised Controlled Trial 


\section{Introduction}

Advances in immunosuppression and patient care following renal transplantation have led to significant reductions in early acute rejection rates with one-year graft survival in excess of $90 \%$. Use of acute rejection, graft survival, or composites of these endpoints in clinical trials is no longer feasible due to the large numbers of participants required to demonstrate significant improvements in outcomes. Measures of early graft function have been suggested to be reliable surrogates for medium to long-term graft survival, and so many trials employ measures of renal function as primary or secondary endpoints (1-4).

Gold-standard for the assessment of renal function remains formal measurement of glomerular filtration rate (GFR) by a reference method such as inulin clearance or radioisotope excretion. However, the time and cost implications of these methods mean that they are usually impractical for use in the clinical trial setting, or indeed for routine clinical use. Estimated glomerular filtration rate (eGFR) or creatinine clearance ( $\mathrm{CrCl})$, using calculations based upon serum creatinine levels, are often used as more practical alternatives, but commonly used eGFR equations have generally been derived from non-transplant patient populations and perform variably in transplant recipients, with high levels of bias and low precision (5).

A previous review of trials in renal transplantation published between 2003 and 2008 demonstrated that the use of renal function endpoints is common, with $28 \%$ studies using renal function as the primary endpoint (6). Use of formal GFR measurements was uncommon, in just $9 \%$ trials, with eGFR preferred in $61 \%$. The most commonly utilised eGFR methods were the Cockroft-Gault (62\%) and Nankivell (23\%) equations.

Since this analysis, a number of studies have demonstrated that these commonly used equations underperform in renal transplant recipients. Whilst the literature suggests that the MDRD equation demonstrates superior performance to alternatives in transplant recipients, it does have limitations. The formula was derived in a population of patients with end stage renal disease of varying stages, with a mean GFR of just under $40 \mathrm{ml} / \mathrm{min} / 1.73 \mathrm{~m}^{2}$ (7). It is known to under-estimate the true GFR in both renal and transplant patents with GFR over $60 \mathrm{ml} / \mathrm{min} / 1.73 \mathrm{~m}^{2}(8,9)$. In an attempt to address this, the Chronic Kidney Disease Epidemiology Collaboration (CKD-EPI) equation was developed that includes a correction for patients with a low serum creatinine level (10), and is now the recommended formula for routine clinical use in patients with chronic kidney disease (11).

In light of the better understanding of the relative benefits of different renal function measures in renal transplant recipients over recent years, along with the development of new equations for the prediction of GFR, this study aims to reassess the variability and quality of reporting of renal function endpoints in trials of renal transplant immunosuppression. 


\section{Methods}

Identification of studies

We searched the Transplant Library database (www.transplantlibrary.com) for all randomised controlled trials comparing immunosuppressive interventions in renal transplant recipients published over a 5-year period between January $1^{\text {st }} 2010$ and December $31^{\text {st }} 2014$. The Transplant Library database is maintained by the Centre for Evidence in Transplantation, and contains all reports from randomised controlled trials in the field of solid organ transplantation published from 1970 to date, sourced from MEDLINE, the Cochrane Library and hand-searches of relevant conference abstracts. Filters were applied for renal transplantation, full text articles (excluding conference abstracts) and date.

Search results were screened independently by two reviewers independently for inclusion in the study. Discrepancies in inclusion were agreed by discussion.

Inclusion/Exclusion Criteria

All full text reports from randomised controlled trials comparing two or more immunosuppressive strategies (induction or maintenance) in adult renal transplant recipients, were eligible for inclusion. Articles relating to non-immunosuppressive interventions, paediatric transplantation, and other organ transplant types or published in a language other than English were excluded. We also excluded those studies not providing any clinical outcome data, including those only reporting pharmacokinetic data. Conference abstracts were excluded due to the lack of complete description of methods making assessment regarding outcome reporting impossible.

\section{Data Extraction}

Relevant demographic data were extracted from all studies to include number of participants, interventions used, number of centres, study design quality, country, funding source and evidence of ethics approval. Quality at a study level was assessed by means of the Jadad score (12), description of adequate allocation concealment and presence of an intention-to-treat analysis.

Data regarding renal function endpoints were extracted in detail, including the endpoint and method of assessment/equation used, time-point at which the outcome was measured, mean and standard deviation in the control and study groups, completeness of reporting and whether a significant difference between treatment arms was reported. Discrepancies in clinical effect between different renal function measures were also documented.

Completeness of outcome reporting

Completeness of outcome reporting was related to whether the information provided would be sufficient for inclusion in meta-analysis. For binary outcomes, this required the number of patients and the denominator for each group to be reported (these could be expressed as percentages or odds ratios as long as confidence intervals were given). For continuous outcomes, the mean and standard deviation (or median and range for 
non-parametric data) as well as the total number of patients in each group were required. Completeness of reporting was classified into several levels: completely reported, reported but missing data (e.g. standard deviation (SD), range, confidence interval), reported but only as significant ( $\mathrm{p} \geq 0.05)$, reported but only as nonsignificant $(p<0.05)$, graphical representation only and mentioned in methods but not reported in the results. Data analysis

All demographic and outcome information was extracted into a custom-designed MySQL database. All data extraction was completed by one author (SH) and validated by a second author (SK) to ensure completeness and accuracy. Any discrepancies in data extraction were agreed by discussion between authors.

Extracted data were exported to the R statistical software and analysed using simple summary statistics. Where more than one measure of renal function was reported in a manuscript, the consistency of findings (whether comparisons were statistically significant or not) was examined. For studies reporting the MDRD eGFR, those reporting a mean eGFR of greater than $60 \mathrm{ml} / \mathrm{min} / 1.73 \mathrm{~m}^{2}$ were identified as the MDRD equation is known to underperform in patients with preserved renal function. 


\section{Results}

Characteristics of included studies

The literature search identified 213 manuscripts from 182 unique studies that met the inclusion criteria (figure 1). Characteristics of the included studies are described in table 1. The median number of centres in each trial was 4 (range 1-111) and the median length of follow up in weeks was 52 (range 1-932). Median number of participants was 115 (range 9-1,640).

$14.8 \%$ studies reported results based on available case analysis, $30.2 \%$ per protocol analysis, $19.7 \%$ intention to treat analysis and $35.2 \%$ modified intention to treat analysis. $48 \%$ studies reported an adequate method of allocation concealment. The median Jadad score was 3 (range 0 to 5).

Reporting of renal function endpoints

Overall, 174 (81.7\%) manuscripts reported at least one renal function endpoint (figure 2). In 44 (20.7\%) renal function was defined as the primary endpoint. There were no significant differences in study characteristics between studies reporting renal function endpoints and those not (table 1).

Completeness of reporting

Of the total of 345 reported endpoints across all studies, $86.1 \%$ were completely reported with summary effect, measures of variance and the number of patients included in analysis (table 2). 3.2\% had missing measures of variance, $3.5 \%$ were only reported as significant/non-significant, $5.8 \%$ had a graphical representation of results only, and in $1.4 \%$ renal function endpoints were mentioned in the methods section of the manuscript but not reported in the results.

Reporting quality of serum creatinine and estimated GFR endpoints were similar. All studies reporting formal GFR measurements reported these endpoints completely with no missing data.

Serum creatinine

103 (48.4\%) manuscripts reported serum creatinine as a marker of graft function. In 22 manuscripts (10.3\%) serum creatinine was the only measure of renal function reported, and in 5 it was reported as the primary endpoint.

Estimated GFR

A measure of estimated GFR was reported in 142 (66.7\%) manuscripts. Formulas used for GFR estimation were MDRD (42.3\%), Cockroft Gault (23.5\%), Nankivell (15.0\%) and CKD-EPI (0.9\%) (figure 2). 6 manuscripts (2.8\%) did not report the formula used to estimate GFR. 26 manuscripts (12.2\%) reported eGFR calculated by more than one method.

Of the manuscripts reporting MDRD eGFR, 29 (32.2\%) reported mean GFR of over $60 \mathrm{ml} / \mathrm{min}$ in at least one group.

Measured GFR 
26 manuscripts (12.2\%) reported mGFR as an endpoint. 14 (6.6\%) reported creatinine clearance and 14 (6.6\%) reported radioisotope measured GFR (EDTA 2, iothalamate 7 and iohexol 5). 2 studies did not report the method used.

Discrepancies between renal function estimates

In ten of the included manuscripts, the statistical significance of the effect of intervention on renal function differed when alternative renal function endpoints were used. In four manuscripts, measures of eGFR disagreed with function measured by serum creatinine alone (13-16), and in three eGFR assessed by different formulae disagreed (14-17). In five of the 26 manuscripts (19.2\%) reporting measured GFR or creatinine clearance, statistical significance differed between the measured GFR/CrCl and estimated GFR or serum creatinine (18-22). Variability in the methodology used to measure function and the formulae used for estimates make meaningful comparisons between mGFR and eGFR estimates within studies impossible. 


\section{Discussion}

The present analysis demonstrates that there is still a great deal of heterogeneity in the reporting of renal function endpoints in clinical trials of immunosuppression following renal transplantation, both in terms of the measures used and in the quality of reporting. Various combinations of serum creatinine, estimated $\mathrm{GFR} / \mathrm{CrCl}$ and measured $\mathrm{GFR} / \mathrm{CrCl}$ are employed, with use of different formulae for estimating function. Studies often use more than one measure to assess function, with some evidence of discrepancies between methods that could affect the conclusions depending on the measure used.

Despite being the gold standard, formal measurements of renal function are only reported in $12 \%$ of the present manuscripts. This reflects the difficulty in formal GFR assessment, in terms of cost, time and patient acceptability, which limits uptake as a trial endpoint. The finding that a significant proportion of studies (19.2\%) measuring GFR have discrepancies between these formal measurements and other estimates of renal function probably reflects the fact that most of these studies have significant amount of missing data for measured GFR. There is therefore a trade-off between measuring a complex outcome with risk of missing data, and using a less-than perfect estimate which can be more reliably collected.

It is interesting that $10 \%$ manuscripts reported serum creatinine as the only measure of renal function, with 5 including it as a primary endpoint. This is contrary to current guidelines, which recommend the use of creatinine-based GFR estimates for routine follow-up in all patients (23). The effect of race, age, gender and muscle mass make serum creatinine an unreliable marker of function, with a highly variable relationship with GFR in transplant recipients (24). This is likely reflected in the present analysis, with discrepancies seen between serum creatinine values and other markers of function.

In their previous analysis, White and colleagues demonstrated that the most commonly used creatinine-based estimates in transplant clinical trials were the Cockroft-Gault and Nankivell equations (6). Given the increasing evidence that the MDRD formula offers improved prediction in the transplant population, it is perhaps not surprising that in the present cohort the MDRD formula is now the most commonly used in over $40 \%$ studies. Despite consistent evidence the the Nankivell formula under-performs compared to other equations, it was still used in around $15 \%$ studies (25).

The newer CKD-EPI formula, designed to replace the MDRD equation, has failed to gain traction as yet in transplant trials with only two of the present studies using this outcome. The formula was designed to correct the under-estimation of GFR at low serum creatinine levels. It is therefore of note that nearly one third of the present studies reporting MDRD eGFR reported a mean eGFR in at least one group of greater than 60 $\mathrm{ml} / \mathrm{min} / 1.73 \mathrm{~m}^{2}$, levels at which the MDRD equation is known to under-perform. Despite the theoretical benefit of the CKD-EPI formula in renal transplant recipients with good graft function, studies comparing it with MDRD have demonstrated very variable results. White et al. compared the two formulae in a cohort of 207 stable kidney transplant recipients, and demonstrated a higher proportion of estimates within $30 \%$ of the true GFR with CKD-EPI (84\% vs. 77\%) (26). In the cohort with GFR greater than $60 \mathrm{ml} / \mathrm{min} / 1.73 \mathrm{~m}^{2}$, bias and accuracy improved further with the CKD-EPI formula. Similar benefit has been demonstrated in a population 
of Thai transplant recipients (27). In contrast, other large studies in stable transplant cohorts have demonstrated no benefit for the CKD-EPI equation over the MDRD formula, with higher accuracy using MDRD $(28,29)$.

There are a number of reasons why eGFR equations may under-perform in renal transplant recipients. Patient level factors can affect accuracy, with baseline GFR, age, body mass index (BMI), gender, acute rejection episodes and infections all affecting the performance of commonly-used equations $(8,24)$. Immunosuppression may also play a role. Kukla and colleagues measured the performance of the Cockroft-Gault, MDRD and CKDEPI equations compared to a reference standard iothalamate GFR in a renal transplant population within 3 months and again at 1 year following transplantation (30). As with previous studies, the eGFR measures all over-estimated the measured GFR and bias did not differ between patients on long-term steroids or not. However, there was a significant drop in precision in those patients receiving long-term maintenance steroids, which may relate to an increase in body fat to muscle ratio in patients receiving long-term steroids (31). Another factor that may affect the reliability of creatinine-based equations in transplant recipients is the use of co-trimoxazole as prophylaxis against pneumocystis jiroveci pneumonia, which has been shown to reduce the renal excretion of creatinine and elevate the serum creatinine level in transplant recipients $(32,33)$.

Given the degree of heterogeneity in methods used for estimation of both eGFR and mGFR in the present cohort of studies, it was not possible to pool or directly compare these estimates. However, we were able to demonstrate that in ten of the included manuscripts, there were discrepancies between the statistical significance of effect of intervention on renal function when different methods were used. These discrepancies suggest that the choice of renal function endpoint could result in different conclusions being made as to the effect of an intervention.

A further finding in the current study is the variable quality of reporting of renal function endpoints. $14 \%$ of studies had missing data for these outcomes which would make inclusion in meta-analysis difficult or impossible. In particular, 5 studies defined renal function endpoints in the methods that were then not reported in the results, leading to risk of reporting bias. 6 manuscripts reported eGFR as an endpoint without defining the formula used, and 2 did not report the method used for formal GFR measurement. Similar findings have been demonstrated in an earlier analysis of trials in renal transplantation with significant variability and risk of reporting bias identified in measures of death, graft loss and renal function (34).

One possible solution to this variability would be to develop a consensus definition for a renal function endpoint for use in transplant clinical trials. This would improve consistency and quality of reporting, facilitating comparison between studies and allowing greater reliability in the combination of studies in metaanalysis. Given the lack of agreement in the literature as to the best measure to use, this may prove challenging, but there are certainly some GFR estimates that underperform in transplant recipients and should be avoided $(5,25)$. Projects to define sets of core outcomes for use in clinical trials, such as the ongoing SONGTx project (http://songinitiative.org/song-tx/), may help to facilitate consensus. 
The strength of the present study is the comprehensive analysis of renal function endpoints in all consecutive reports of randomised controlled trials of immunosuppressive interventions in renal transplant recipients over a five-year period. There are however some limitations. Assessment of the impact of discrepancies between different methods of renal function reporting is limited to those studies that reported more than one measure of function, and may not be representative. It is also not possible to assess the true risk of reporting bias without referring to the original study protocols (if available). For example, not all studies reporting eGFR also reported serum creatinine values as endpoints, despite the fact that they must have been measured in order for the eGFR to be estimated. Finally, the included studies were all reported in the period between 2010 and 2014, but the study protocols were designed earlier. It is possible that contemporary studies make greater use of new equations, such as the CKD-EPI formula. The only way to assess this for current studies would be to examine entries in trial registries, the consistency of which varies considerably (35).

In summary, the present analysis demonstrates that the previously reported variability in renal function reporting in transplant clinical trials remains, although there has been a significant change in the formulas used to estimate GFR over the past ten years. There is still some evidence that estimates of GFR are being used inappropriately, and discrepancies between renal function endpoints may affect the conclusions made. Quality and completeness of reporting remains suboptimal and can be improved. Consensus as to the optimal, practical, renal function endpoint for use in transplant clinical trials would greatly improve quality and consistency in reporting.

\section{Declaration of conflicts of interest}

Neither author has any conflict of interest to declare as defined in the instructions to authors. The contents of this manuscript have not been published previously, in part or as a whole. Preliminary results from this project were presented as a poster at the British Transplantation Society Annual Congress 2016. 


\section{References}

1. First MR. Renal function as a predictor of long-term graft survival in renal transplant patients. Nephrology, Dialysis, Transplantation: Official Publication of the European Dialysis and Transplant Association - European Renal Association 2003; 18 Suppl 1: i3.

2. Kasiske BL, Israni AK, Snyder JJ, Skeans MA, Patient Outcomes in Renal Transplantation (PORT) Investigators. The relationship between kidney function and long-term graft survival after kidney transplant. American Journal of Kidney Diseases: The Official Journal of the National Kidney Foundation 2011; 57: 466.

3. Salvadori M, Rosati A, Bock A et al. Estimated one-year glomerular filtration rate is the best predictor of long-term graft function following renal transplant. Transplantation 2006; 81: 202.

4. Schnitzler MA, Lentine KL, Axelrod D et al. Use of 12-month renal function and baseline clinical factors to predict long-term graft survival: application to BENEFIT and BENEFIT-EXT trials. Transplantation 2012; 93: 172.

5. White CA, Huang D, Akbari A, Garland J, Knoll GA. Performance of creatinine-based estimates of GFR in kidney transplant recipients: a systematic review. American Journal of Kidney Diseases: The Official Journal of the National Kidney Foundation 2008; 51: 1005.

6. White CA, Siegal D, Akbari A, Knoll GA. Use of kidney function end points in kidney transplant trials: a systematic review. American Journal of Kidney Diseases: The Official Journal of the National Kidney Foundation 2010; 56: 1140.

7. Levey AS, Bosch JP, Lewis JB, Greene T, Rogers N, Roth D. A more accurate method to estimate glomerular filtration rate from serum creatinine: a new prediction equation. Modification of Diet in Renal Disease Study Group. Annals of Internal Medicine 1999; 130: 461.

8. Bosma RJ, Doorenbos CRC, Stegeman CA, Van Der Heide JJH, Navis G. Predictive Performance of Renal Function Equations in Renal Transplant Recipients: An Analysis of Patient Factors in Bias. American Journal of Transplantation 2005; 5: 2193.

9. Stevens LA, Coresh J, Feldman HI et al. Evaluation of the Modification of Diet in Renal Disease Study Equation in a Large Diverse Population. Journal of the American Society of Nephrology 2007; 18: 2749.

10. Levey AS, Stevens LA, Schmid CH et al. A new equation to estimate glomerular filtration rate. Annals of Internal Medicine 2009; 150: 604.

11. Kidney Disease: Improving Global Outcomes (KDIGO) CKD Work Group. KDIGO 2012 Clinical Practice Guideline for the Evaluation and Management of Chronic Kidney Disease. Kidney International 2013; 3: 1.

12. Jadad AR, Moore RA, Carroll D et al. Assessing the quality of reports of randomized clinical trials: is blinding necessary? Controlled Clinical Trials 1996; 17: 1.

13. Bechstein WO, Paczek L, Wramner L, Squifflet J-P, Zygmunt AJ, European Rapamune Tacrolimus Study Group. A comparative, randomized trial of concentration-controlled sirolimus combined with reduceddose tacrolimus or standard-dose tacrolimus in renal allograft recipients. Transplantation Proceedings 2013; 45: 2133.

14. Lin S, Henning AK, Akhlaghi F, Reisfield R, Vergara-Silva A, First MR. Interleukin-2 receptor antagonist therapy leads to increased tacrolimus levels after kidney transplantation. Therapeutic Drug Monitoring 2015; 37: 206. 
15. Friman S, Arns W, Nashan B et al. Sotrastaurin, a novel small molecule inhibiting protein-kinase C: randomized phase II study in renal transplant recipients. American Journal of Transplantation: Official Journal of the American Society of Transplantation and the American Society of Transplant Surgeons 2011; 11: 1444.

16. Tedesco Silva H, Cibrik D, Johnston T et al. Everolimus plus reduced-exposure CsA versus mycophenolic acid plus standard-exposure CsA in renal-transplant recipients. American Journal of Transplantation: Official Journal of the American Society of Transplantation and the American Society of Transplant Surgeons 2010; 10: 1401.

17. Silva HT, Yang HC, Meier-Kriesche H-U et al. Long-term follow-up of a phase III clinical trial comparing tacrolimus extended-release/MMF, tacrolimus/MMF, and cyclosporine/MMF in de novo kidney transplant recipients. Transplantation 2014; 97: 636.

18. Heilman RL, Younan K, Wadei HM et al. Results of a prospective randomized trial of sirolimus conversion in kidney transplant recipients on early corticosteroid withdrawal. Transplantation 2011; 92: 767.

19. Mjörnstedt L, Sørensen SS, von Zur Mühlen B et al. Improved renal function after early conversion from a calcineurin inhibitor to everolimus: a randomized trial in kidney transplantation. American Journal of Transplantation: Official Journal of the American Society of Transplantation and the American Society of Transplant Surgeons 2012; 12: 2744.

20. Refaie AF, Mahmoud KM, Ismail AM, Sheashaa HA, Kamal AI, Ghoneim MA. Alemtuzumab preconditioning allows steroid-calcineurin inhibitor-free regimen in live-donor kidney transplant. Experimental and Clinical Transplantation: Official Journal of the Middle East Society for Organ Transplantation 2011; 9: 295.

21. Song $\mathrm{Y}, \mathrm{Xue} \mathrm{W}$, Tian $\mathrm{P}$ et al. Combination therapy with diltiazem plus CsA/MMF/Pred or CsA/Aza/Pred triple immunosuppressive regimens for use in clinical kidney transplantation in Northwestern China. European Journal of Clinical Pharmacology 2011; 67: 553.

22. Weir MR, Mulgaonkar S, Chan L et al. Mycophenolate mofetil-based immunosuppression with sirolimus in renal transplantation: a randomized, controlled Spare-the-Nephron trial. Kidney International 2011; 79: 897.

23. Kidney Disease: Improving Global Outcomes (KDIGO) Transplant Work Group. KDIGO clinical practice guideline for the care of kidney transplant recipients. American Journal of Transplantation: Official Journal of the American Society of Transplantation and the American Society of Transplant Surgeons 2009; 9 Suppl 3: S1.

24. Nankivell BJ, Gruenewald SM, Allen RD, Chapman JR. Predicting glomerular filtration rate after kidney transplantation. Transplantation 1995; 59: 1683.

25. Shaffi K, Uhlig K, Perrone RD et al. Performance of creatinine-based GFR estimating equations in solidorgan transplant recipients. American Journal of Kidney Diseases: The Official Journal of the National Kidney Foundation 2014; 63: 1007.

26. White CA, Akbari A, Doucette S, Fergusson D, Knoll GA. Estimating glomerular filtration rate in kidney transplantation: is the new chronic kidney disease epidemiology collaboration equation any better? Clinical Chemistry 2010; 56: 474.

27. Townamchai N, Praditpornsilpa K, Chawatanarat T et al. The validation of estimated glomerular filtration rate (eGFR) equation for renal transplant recipients. Clinical Nephrology 2013; 79: 206.

28. Masson I, Flamant M, Maillard N et al. MDRD versus CKD-EPI equation to estimate glomerular filtration rate in kidney transplant recipients. Transplantation 2013; 95: 1211. 
29. Pöge U, Gerhardt T, Stoffel-Wagner B, Sauerbruch T, Woitas RP. Validation of the CKD-EPI formula in patients after renal transplantation. Nephrology Dialysis Transplantation 2011; 26: 4104.

30. Kukla A, El-Shahawi Y, Leister E et al. GFR-estimating models in kidney transplant recipients on a steroidfree regimen. Nephrology, Dialysis, Transplantation: Official Publication of the European Dialysis and Transplant Association - European Renal Association 2010; 25: 1653.

31. Dolgos S, Hartmann A, Jenssen T, Isaksen GA, Pfeffer P, Bollerslev J. Determinants of short-term changes in body composition following renal transplantation. Scandinavian Journal of Urology and Nephrology 2009; 43: 76.

32. Berglund F, Killander J, Pompeius R. Effect of trimethoprim-sulfamethoxazole on the renal excretion of creatinine in man. The Journal of Urology 1975; 114: 802.

33. Dijkmans BA, van Hooff JP, de Wolff FA, Mattie $\mathrm{H}$. The effect of co-trimoxazole on serum creatinine. British Journal of Clinical Pharmacology 1981; 12: 701.

34. Masson P, Duthie FA, Ruster LP et al. Consistency and completeness of reported outcomes in randomized trials of primary immunosuppression in kidney transplantation. American Journal of Transplantation: Official Journal of the American Society of Transplantation and the American Society of Transplant Surgeons 2013; 13: 2892.

35. Dwan K, Altman DG, Cresswell L, Blundell M, Gamble CL, Williamson PR. Comparison of protocols and registry entries to published reports for randomised controlled trials. The Cochrane Database of Systematic Reviews 2011; : MR000031. 


\section{Tables and Figures}

Figure 1 - Flow chart to demonstrate inclusion/exclusion of studies during the review process. RCT randomised controlled trial.

Figure 2 - Frequency of reporting of renal function endpoints in randomised controlled trials of immunosuppressive interventions. GFR - glomerular filtration rate; MDRD - modification of diet in renal disease; CKD-EPI - Chronic Kidney Disease Epidemiology Collaboration. 
Table 1 - Characteristics and study-level quality assessment of included studies.

\begin{tabular}{|c|c|c|}
\hline Characteristic & $\begin{array}{l}\text { All studies } \\
(\mathrm{N}, \%)\end{array}$ & $\begin{array}{l}\text { Studies reporting renal } \\
\text { function endpoints ( } N, \%)\end{array}$ \\
\hline \multicolumn{3}{|l|}{ Recipient type } \\
\hline De novo & $128(70.3)$ & $106(71.6)$ \\
\hline Post-transplant & $54(29.7)$ & $42(28.4)$ \\
\hline \multicolumn{3}{|l|}{ Donor type } \\
\hline Deceased & $28(15.4)$ & $23(15.5)$ \\
\hline Living & $24(13.2)$ & $20(13.5)$ \\
\hline Either & $130(71.4)$ & 105 (70.9) \\
\hline \multicolumn{3}{|l|}{ Number of centres } \\
\hline Single & $80(44.0)$ & $63(42.6)$ \\
\hline Multiple & $102(56.0)$ & $85(57.4)$ \\
\hline \multicolumn{3}{|l|}{ Ethics approval } \\
\hline Yes & 160 (87.9) & 133 (89.9) \\
\hline No & $22(12.1)$ & $15(10.1)$ \\
\hline \multicolumn{3}{|l|}{ Funding source } \\
\hline Industry & $99(54.4)$ & $80(54.1)$ \\
\hline Non-industry & $34(18.7)$ & $28(18.9)$ \\
\hline Mixed & $16(8.8)$ & $14(9.5)$ \\
\hline No external funding & $7(3.8)$ & $6(4.1)$ \\
\hline Not described & $26(14.3)$ & $19(12.8)$ \\
\hline \multicolumn{3}{|l|}{ Jadad score } \\
\hline 0 & $1(0.5)$ & $1(0.7)$ \\
\hline 1 & $21(11.5)$ & $14(9.5)$ \\
\hline 2 & $67(36.8)$ & $60(40.5)$ \\
\hline 3 & $83(45.6)$ & $65(43.9)$ \\
\hline 4 & $2(1.1)$ & $2(1.3)$ \\
\hline 5 & $8(4.4)$ & $6(4.1)$ \\
\hline \multicolumn{3}{|l|}{ Adequate allocation concealment } \\
\hline No & $95(52.2)$ & $79(53.4)$ \\
\hline Yes & $87(47.8)$ & $69(46.6)$ \\
\hline \multicolumn{3}{|l|}{ Intention-to-treat analysis } \\
\hline Available case analysis & $27(14.8)$ & $21(14.2)$ \\
\hline Intention to treat & $36(19.7)$ & 31 (20.9) \\
\hline Modified intention to treat & $64(35.2)$ & $53(35.8)$ \\
\hline Per Protocol analysis & $55(30.2)$ & $43(29.1)$ \\
\hline
\end{tabular}


Table 2 - Completeness of reporting for renal function endpoints. GFR - glomerular filtration rate; $\mathrm{CrCl}$ - $\mathrm{creatinine} \mathrm{clearance.}$

\begin{tabular}{|c|c|c|c|c|c|c|c|}
\hline \multirow[t]{2}{*}{ Endpoint } & \multirow[t]{2}{*}{ Total (N) } & \multicolumn{6}{|c|}{ Completeness of Reporting ( $\mathbf{N}, \%)$} \\
\hline & & Complete & $\begin{array}{l}\text { Missing measure of } \\
\text { variance }\end{array}$ & $\begin{array}{l}\text { Reported as } \\
\text { significant } \\
(P<0.05)\end{array}$ & $\begin{array}{l}\text { Reported as } \\
\text { non-significant } \\
(p>0.05)\end{array}$ & $\begin{array}{l}\text { Graphical } \\
\text { representation }\end{array}$ & $\begin{array}{l}\text { In methods but not } \\
\text { reported }\end{array}$ \\
\hline Overall & 345 & $297(86.1)$ & $11(3.2)$ & $3(0.8)$ & $9(2.6)$ & $20(5.8)$ & $5(1.4)$ \\
\hline Serum Creatinine & 107 & $87(81.3)$ & $4(3.7)$ & $0(0)$ & $4(3.7)$ & $8(7.5)$ & $4(3.7)$ \\
\hline Estimated GFR/CrCl & 201 & $173(86.1)$ & $7(3.5)$ & $3(1.5)$ & $5(2.5)$ & $12(6.0)$ & $1(0.5)$ \\
\hline Measured GFR/CrCl & 35 & $35(100)$ & $0(0)$ & $0(0)$ & $0(0)$ & $0(0)$ & $0(0)$ \\
\hline
\end{tabular}

